\title{
Neutron surface scattering. Application to magnetic thin films
}

\author{
Frédéric Ott
}

Laboratoire Léon Brillouin, UMR12 CEA-CNRS, Centre d'Etudes de Saclay, 91191 Gif-sur-Yvette, France

\section{INTRODUCTION}

Our objective is to characterize surfaces and interfaces at the nanometric scale. This corresponds to very small volumes of matter of the order of a few micrograms. Thus, it is necessary to develop surface scattering techniques. The use of neutron reflection at grazing incidence increases the neutron interaction with the sample surface and makes such experiments feasible.

It is possible to distinguish three grazing incidence scattering geometries (see Fig. 1): specular reflection, scattering in the incidence plane (off-specular scattering) and scattering perpendicular to the incidence plane. These different scattering geometries allow to probe different length scales $\xi$ and directions in the sample surface. Specular reflectivity (red line) allows to probe the structure along the depth in the film $(3 \mathrm{~nm} \leq \xi \leq 100 \mathrm{~nm})$. Off-specular scattering measurements (blue plane) allows to probe in-plane surface features at a micrometric scale $(600 \mathrm{~nm} \leq \xi \leq 60 \mu \mathrm{m})$. GISANS Grazing Incidence SANS (green plane) allows to probe in-plane surface features in the range $3 \mathrm{~nm} \leq \xi \leq$ $100 \mathrm{~nm}$. These different scattering geometries allow to probe a very wide range of length-scales $\xi$, ranging from a few nm up to several $\mu \mathrm{m}$. Structures perpendicular and in-plane can be probed.

In this chapter we will restrict ourselves to specular reflectivity. We will present the principle of the technique and some examples of measurements on real multilayer systems.

\section{SPECULAR REFLECTIVITY}

\subsection{Neutron reflection}

Let $\mathrm{Q}$ be the scattering wave-vector defined by:

$$
Q=k_{r}-k_{i}=\frac{4 \pi}{\lambda} \sin \theta
$$

where $\lambda$ is the incident neutron wavelength and $\theta$ is the incidence angle of the neutron beam on the surface (see Fig. 2a). In this geometry, the reflection angle is equal to the incidence angle, the scattering wave vector $\mathrm{Q}$, is perpendicular to the sample surface.

If one considers a surface, it is basically formed by atoms (see Fig. 2b). However, since the incidence angles are very small, of the order of a few degrees, the scattering wave-vector $\mathrm{Q}$ is of the order of a few $\mathrm{nm}^{-1}$ (as deduced from Eq. (1)). That is, we are probing length-scales of the order of a few nanometres 


\section{Collection SFN}

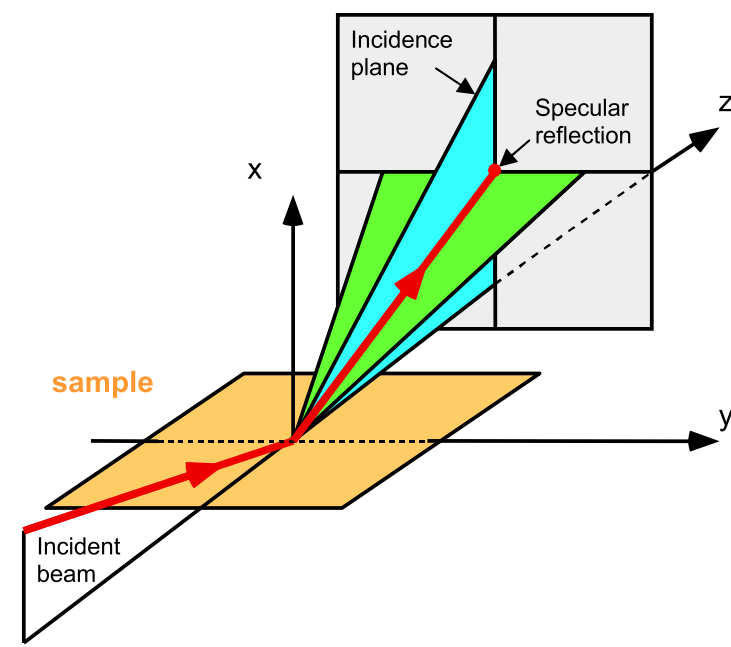

Specular reflection

$0.06<\mathrm{Q}_{\mathrm{z}}<3 \mathrm{~nm}^{-1}$

$3 \mathrm{~nm}<\xi<100 \mathrm{~nm}$

Incidence plane

$10^{-4}<\mathrm{Q}_{\mathrm{x}}<10^{-2} \mathrm{~nm}^{-1}$

$600 \mathrm{~nm}<\xi<60 \mu \mathrm{m}$

Plane perpendicular

to the incidence plane

$10^{-4}<\mathrm{Q}_{\mathrm{y}}<3 \mathrm{~nm}^{-1}$

$3 \mathrm{~nm}<\xi<100 \mathrm{~nm}$

Figure 1. The different surface scattering geometries. (red) specular reflectivity geometry; (blue) off-specular scattering plane, corresponding to the incidence plane; (green) GISANS scattering plane, perpendicular to the incidence plane. These different scattering geometries allow to probe a very wide range of length-scales and direction in the sample surface.
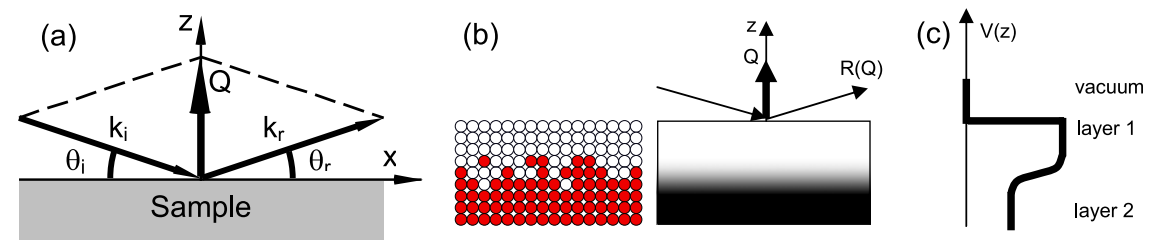

Figure 2. (a) Specular reflectivity geometry. The reflection angle is equal to the incidence angle; the scattering wave-vector $\mathrm{Q}$ is perpendicular to the sample surface. (b) Interface between 2 surfaces. In the optical approximation, the interface is approximated as a continuous medium. (c) Reflection on a thin film deposited on a surface. The reflectivity measures the Fourier transform of the interaction potential V(z).

and thus the atomics details of the structure are invisible. We can apply the optical approximation and consider the material as a continuous medium. We introduce the scattering length density (SLD), which is given by:

$$
\mathrm{SLD}=b_{V}=\frac{1}{V} \sum_{i \in V} b_{i}
$$

where $b_{i}$ is the scattering length of the atomic species $\mathrm{i}$ and $\mathrm{V}$ is a volume over which the structure can be averaged (typically one atomic cell). In the case of a crystalline structure, the SLD can be calculated as $\operatorname{SLD}=\rho b$ where $\rho$ is the atomic density in $m^{-3}$ and $b$ is the scattering length of the atom (in $m$ ).

In a specular reflection geometry, we also make the assumption that the system is invariant by translation along the sample surface so that there are contributions to the scattering only from the sample structure along the $(\mathrm{Oz})$ direction. Figure $2 \mathrm{c}$ illustrates the way an interface is modeled in a reflectivity experiment. The SLD is only a function of the depth $\mathrm{z}$ in the film: $\operatorname{SLD}(\mathrm{z})$. 


\subsection{What information is obtained in a reflectivity measurement?}

The neutron interaction potential is given by:

$$
V(z)=\frac{h^{2}}{2 \pi m_{n}} \operatorname{SLD}(z)
$$

where $\operatorname{SLD}(\mathrm{z})$ is the Scattering Length Density along the $(\mathrm{Oz})$ direction. Let $\mathrm{R}(\mathrm{Q})$ be the reflected intensity as a function of the incidence wave-vector. In the Born approximation, the specular reflectivity measures the Fourier transform of the interaction potential profile $\mathrm{V}(\mathrm{z})$ along the direction perpendicular to the sample surface:

$$
R(Q) \propto \mathrm{FT}(V(z))
$$

However, at grazing incidence, the neutron beam can be totally reflected and thus the Born approximation fails. It is thus necessary to perform a fully dynamical calculation.

\subsection{Derivation of the reflectivity}

The neutron behaviour is described by the Schrödinger equation:

$$
\frac{-\hbar^{2}}{2 m_{n}} \Delta \varphi+V \varphi=E \varphi
$$

where $\phi$ is the neutron wave-function, $\mathrm{E}$ is the neutron kinetic energy, $\mathrm{V}$ is the interaction potential (see Eq. (3)). This equation can be expressed as a Helmholtz propagation equation:

$$
\Delta U+k^{2} U=0
$$

where the propagation wave-vector in the medium of potential $\mathrm{V}$ is given by

$$
k^{2}=\frac{2 m_{n}}{\hbar^{2}}(E-V)
$$

\subsubsection{Optical index}

Since the neutron follows a classical propagation equation, it is possible to define an optical index $\mathrm{n}$ in the medium:

$$
n^{2}=\frac{k^{2}}{k_{0}^{2}}
$$

where $k_{0}$ is the wave-vector in vacuum. This optical index is given by:

$$
n^{2}=1-\frac{V}{E}=1-\frac{\lambda^{2}}{\pi} \mathrm{SLD}
$$

which can be approximated with a great accuracy by:

$$
n \approx 1-\frac{\lambda^{2}}{2 \pi} \mathrm{SLD}
$$

At an interface, the neutron reflection follows the classical Snell law (see Fig. 3):

$$
\cos \theta_{i}=n \cos \theta_{t r} .
$$

One important point is that the optical index is generally smaller than one, which means that below a critical angle $\theta_{c}$ there is total reflection (defined by the condition $\theta_{t r}=0$ ). The critical angle is thus 


\section{Collection SFN}

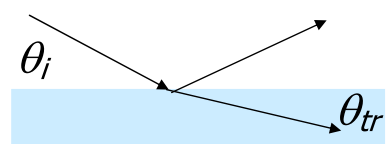

Figure 3. Refraction of a neutron beam at an interface.

Table 1. Nuclear and magnetic optical index $n=1-\delta \pm \delta_{M}$ for some materials at $\lambda=0.4 \mathrm{~nm}$.

\begin{tabular}{|c|r|r|r|}
\hline Element & $\delta\left(\times 10^{-6}\right)$ & $\delta_{M}\left(\times 10^{-6}\right)$ & $\sigma_{a}$ (barns) \\
\hline \hline $\mathrm{H}_{2} \mathrm{O}$ & -1.43 & & \\
\hline $\mathrm{D}_{2} \mathrm{O}$ & 16.2 & & \\
\hline $\mathrm{Si}$ & 5.28 & & \\
\hline $\mathrm{Al}_{2} \mathrm{O}_{3}$ & 14.5 & & \\
\hline polystyren-H & 3.6 & & \\
\hline polystyren-D & 16.5 & & \\
\hline $\mathrm{Au}$ & 11.5 & & \\
\hline $\mathrm{Cu}$ & 16.6 & & \\
\hline $\mathrm{Fe}$ & 20.45 & 11.7 & 2.56 \\
\hline $\mathrm{Co}$ & 5.7 & 10.3 & 37.2 \\
\hline $\mathrm{Ni}$ & 24 & 3.7 & 4.49 \\
\hline $\mathrm{Gd}$ & 5.0 & 14.5 & 49700 \\
\hline
\end{tabular}

given by the equation:

$$
n=\cos \theta_{c}
$$

The expression of the critical angle is:

$$
\theta_{c}=\sqrt{\frac{S L D}{\pi}} \lambda .
$$

The corresponding critical wave-vector is given by:

$$
Q_{c}=4 \sqrt{\pi \mathrm{SLD}} .
$$

For practical purposes, it is convenient to express the optical index as:

$$
n \approx 1-\delta=1-\frac{\lambda^{2}}{2 \pi} \mathrm{SLD}
$$

where $\delta$ is a small deviation from one. Be careful that the optical index is a function of the wavelength and thus the best characterization of a material is given by the SLD. Table 1 summarizes the optical index for some of the most common materials.

One can note that Ni has the highest optical index among all materials. A useful value to remember is that the critical angle of total reflection for $\mathrm{Ni}$ is $0.1 \%$.

\subsubsection{Reflection on a substrate}

As an example, we will now derive the calculation of the reflection of a neutron beam on a simple surface (semi-infinite medium).

Let's consider an incidence plane wave:

$$
\Psi(r)=e^{i k \cdot r}=e^{i k_{/ /} \cdot x} e^{i k_{\perp} \cdot z}=e^{i k_{/ /} \cdot x} \psi(z) .
$$




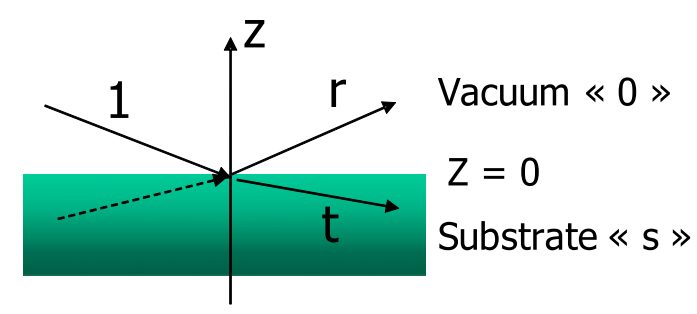

Figure 4. Reflection of a neutron beam on a semi-infinite medium. The amplitudes of the incident, reflected and transmitted waves are respectively $1, \mathrm{r}, \mathrm{t}$.

The longitudinal component of the wave-vector is preserved during the reflection so that the neutron wave-function can be expressed as a z only function. In each medium, there is a wave traveling "upward" and "downward". The neutron wave-function can be expressed by

$$
\psi(z)=A e^{\left(i k_{\perp} z\right)}+B e^{\left(-i k_{\perp} z\right)} .
$$

In the vacuum and in the substrate we have the following equations:

$$
\left\{\begin{array}{l}
\psi_{0}(z)=1 . e^{\left(i k_{\perp 0} z\right)}+r . e^{\left(-i k_{\perp 0} z\right)} \\
\psi_{s}(z)=t . e^{\left(i k_{\perp s} z\right)}+0 . e^{\left(-i k_{\perp s} z\right)}
\end{array}\right.
$$

where " 1 " is the amplitude of the incident wave, " $r$ " is the amplitude of the reflected wave and " $t$ " is the amplitude of the transmitted wave (see Fig. 6). There is no wave coming from underneath the substrate. In order to determine the unknown amplitudes " $\mathrm{r}$ " and "t" we need 2 equations. These equations are given by the continuity equations of the neutron wave function at the interface $z=0$.

$$
\left\{\begin{array}{l}
\psi_{0}(z=0)=\psi_{s}(z=0) \\
\psi_{0}^{\prime}(z=0)=\psi_{s}^{\prime}(z=0)
\end{array}\right.
$$

which, using (19) can be expressed as:

$$
\left\{\begin{array}{l}
1+r=t \\
k_{\perp 0}(1-r)=k_{\perp s} t .
\end{array}\right.
$$

Both amplitudes " $\mathrm{r}$ " and " $\mathrm{t}$ " can then be expressed as:

$$
r=\frac{k_{\perp 0}-k_{\perp s}}{k_{\perp 0}+k_{\perp s}} \quad t=\frac{2 k_{\perp 0}}{k_{\perp 0}+k_{\perp s}} .
$$

The measured reflectivity signals are the square of the above amplitudes. The physically measured signals are:

$$
R=|r|^{2} \text { et } T=|t|^{2} \text {. }
$$

\subsubsection{Reflectivity on non magnetic systems}

The reflectivity on a substrate can easily be computed. Below the critical wave vector $Q_{c}$, the neutrons are totally reflected: $\mathrm{R}=1$. Above approximately $3 . Q_{c}$, the reflectivity decays as $1 / Q^{4}$ (following the classical Born approximation).

In the case of a film deposited on a substrate, oscillations appear in the reflectivity curve. They are due the constructive and destructive interferences between the waves partially reflected at the 2 interfaces (see Fig. 6). The period of the oscillations corresponds to the thickness of the layer: $d=2 \pi / \Delta q$. These fringes are often referred to as Kiessig fringes. 


\section{Collection SFN}

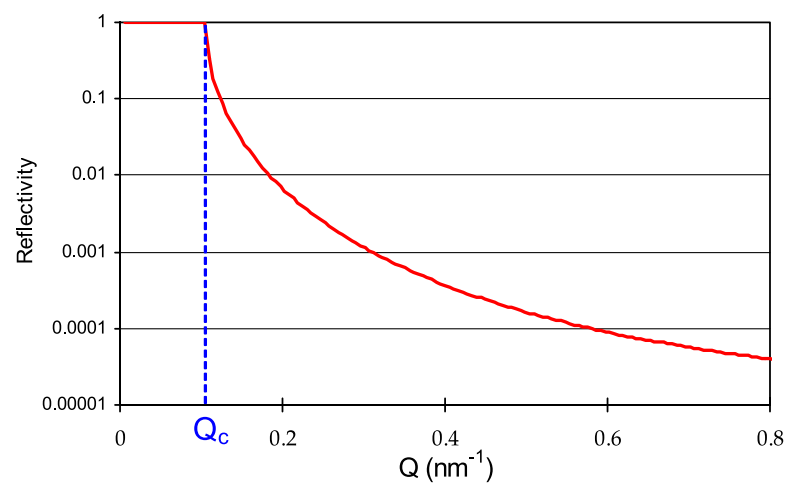

Figure 5. Reflectivity on a semi-infinite medium, below a critical wave-vector $Q_{c}$, there is total reflection, above this point, the signal decays as $1 / Q^{4}$.

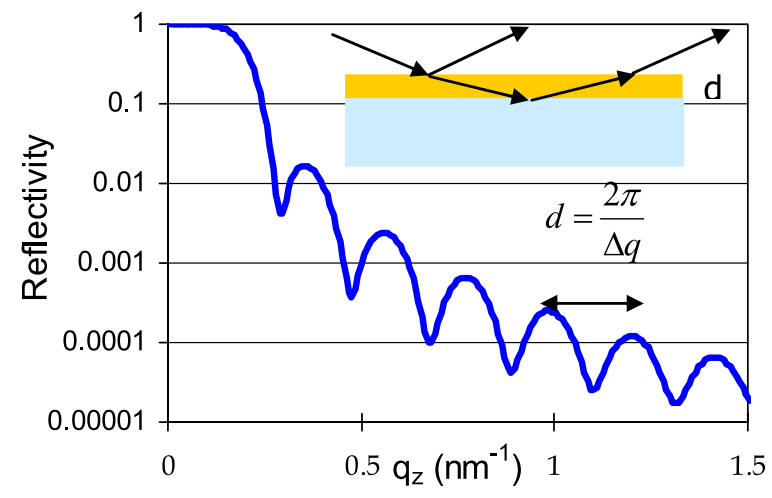

Figure 6. Reflectivity on a Cu film ( $30 \mathrm{~nm})$ deposited on a silicon substrate.

When the multilayer is more complicated, the interference pattern becomes more intricate and numerical modeling is required. Figure 7 illustrates the case of a $\mathrm{Cu}$ layer covered with a thin protective layer of chromium. The top capping layer introduces a long range modulation on top of the interference fringes due to the $\mathrm{Cu}$ layer.

\section{POLARIZED NEUTRON REFLECTIVITY}

\subsection{Magnetic neutron interaction}

Besides the nuclear interaction of the neutron and the nuclei, there is a direct interaction between the neutron spin and a magnetic induction. This interaction is simply given by the Zeeman interaction:

$$
V_{M}(r)=-\vec{\mu}_{n} \cdot \vec{B}(r)
$$

where $\vec{\mu}_{n}$ is the neutron magnetic moment and $\vec{B}(r)$ is the magnetic induction. In a continuous magnetic thin film, the magnetic field in the layer is given by:

$$
B=\mu_{0}\left(H_{0}+(1-D) M\right) .
$$




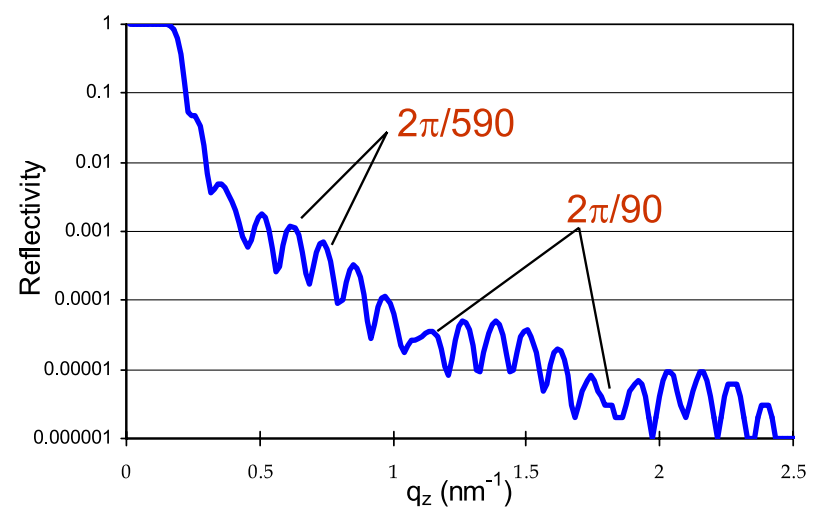

Figure 7. Reflectivity of a bilayer $\mathrm{Cu}(50 \mathrm{~nm}) / \mathrm{Cr}(9 \mathrm{~nm})$ on silicon.

Table 2. Comparison of the nuclear and magnetic scattering lengths for the most common magnetic elements.

\begin{tabular}{|c|c|c|}
\hline Elements & $b_{n}(\mathrm{fm})$ & $b_{M}(\mathrm{fm})$ \\
\hline \hline $\mathrm{Fe}$ & 9.45 & 5.4 \\
\hline $\mathrm{Co}$ & 2.49 & 4.5 \\
\hline $\mathrm{Ni}$ & 10.3 & 1.6 \\
\hline $\mathrm{Gd}$ & $6.5-\mathrm{i} 13.82$ & 18.83 \\
\hline $\mathrm{Si}$ & 4.15 & - \\
\hline
\end{tabular}

where $H_{0}$ is the applied magnetic field, $M$ is the magnetization of the thin film and $D$ is the demagnetizing factor. In a thin film:

$$
(1-D) M=M_{/ /}
$$

where $M_{/ /}$is the projection of the magnetization on the plane of the film. This is a limitation of PNR: the technique is only sensitive to the in-plane component of the magnetization. The total neutron matter interaction potential is thus given by:

$$
V=\frac{h^{2}}{2 \pi m} \mathrm{SLD}-\mu_{n} \cdot \mu_{0} M-\mu_{n} \cdot B_{0} .
$$

In order to compare the nuclear and magnetic interactions, it is possible to express the interaction potential in the form:

$$
V=\frac{h^{2}}{2 \pi m} \rho\left(b_{n}+b_{M}\right) \text { with } b_{m}=\frac{2 \pi m}{h^{2}} \frac{\mu_{n} \mu_{0} M_{/ /}}{\rho}
$$

where $\rho$ is the atomic density.

Table 2 shows the comparison of the nuclear and magnetic scattering lengths for the most common magnetic elements. One can immediately notice that both interactions are of the same order of magnitude. In the case of $\mathrm{Fe}$, the magnetic scattering length amounts up to $50 \%$ of the nuclear scattering length. In the case of $\mathrm{Co}$, the scattering length is twice as big as the nuclear interaction.

\subsection{Reflectivity on a magnetic system}

In the following, we will refer to "up" (resp. "down") for neutrons whose polarisation is parallel (resp. anti-parallel) to the applied magnetic field (which defines the quantization axis). The neutron 


\section{Collection SFN}

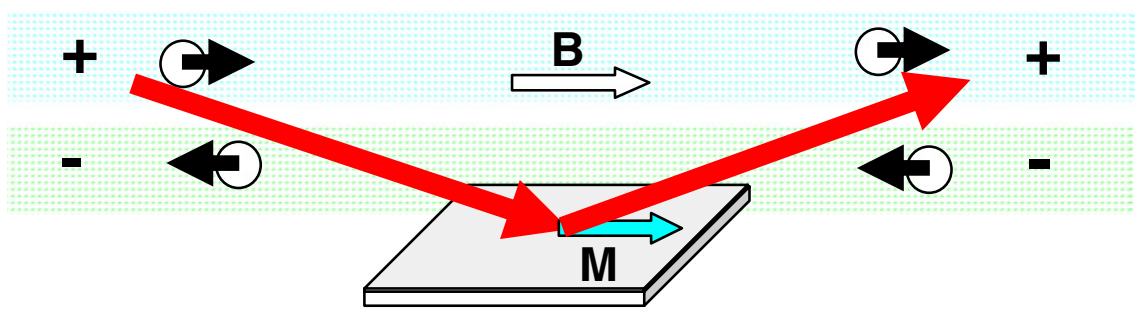

Figure 8. In the case of a magnetization parallel to the applied magnetic field, it is possible to measure 2 different cross sections $R^{++}$and $R^{--}$.

wave-function is expressed as a spinor:

$$
\varphi=a|+\rangle+b|-\rangle=\left(\begin{array}{l}
a \\
b
\end{array}\right) \begin{aligned}
& |+\rangle \\
& |-\rangle
\end{aligned} .
$$

In the case of a magnetic thin film system, the system is birefringent. The optical index depends on the neutron polarization with respect to the magnetization. The optical index is expressed as:

$$
n^{ \pm} \approx 1-\delta \pm \delta_{M}=1-\frac{\lambda^{2}}{2 \pi} \operatorname{SLD} \pm \frac{m \lambda^{2}}{h^{2}} \mu \cdot B
$$

where $n^{+}$is the optical index for "up" neutrons and $n^{-}$is the optical for "down" neutrons. In a classical PNR experiment, it is possible to measure 4 cross sections:

$$
R^{++}, R^{--}, R^{+-}, R^{-+}
$$

- $R^{++}$corresponding to the reflected amplitude of an incident neutron beam neutron "up" and a measurement of the fraction of "up" neutron after reflection (i.e. without spin-flip during the reflection.)

- $R^{+-}$corresponding to the reflected amplitude of an incident neutron beam neutron "up" and a measurement of the fraction of "down" neutron after reflection (i.e. the fraction of the beam which has experienced a spin-flip during the reflection).

The general transfer matrix of the sample is thus:

$$
\varphi_{r}=\left(\begin{array}{ll}
R^{++} & R^{+-} \\
R^{-+} & R^{--}
\end{array}\right) \varphi_{i}
$$

We can distinguish 2 extreme cases.

\subsubsection{Magnetization parallel to the applied magnetic field}

During reflection, the neutrons do not experience any spin-flip. They remain in their eigenstate. The sample transfer matrix is of the form:

$$
\left(\begin{array}{cc}
R^{+} & 0 \\
0 & R^{-}
\end{array}\right)
$$

For an "up" (resp. "down") polarised beam, $\varphi=1|+\rangle($ resp. $\varphi=1|-\rangle)$

$$
\begin{gathered}
{\left[\begin{array}{c}
R^{+} \\
0
\end{array}\right]=\left(\begin{array}{c}
R^{+}, 0 \\
0, R^{-}
\end{array}\right)\left[\begin{array}{l}
1 \\
0
\end{array}\right] \begin{array}{l}
|+\rangle \\
|-\rangle
\end{array} \text { and }\left[\begin{array}{c}
0 \\
R^{-}
\end{array}\right]=\left(\begin{array}{l}
R^{+}, 0 \\
0, R^{-}
\end{array}\right)\left[\begin{array}{l}
0 \\
1
\end{array}\right]|+\rangle} \\
\text { 02004-p.8 }
\end{gathered}
$$



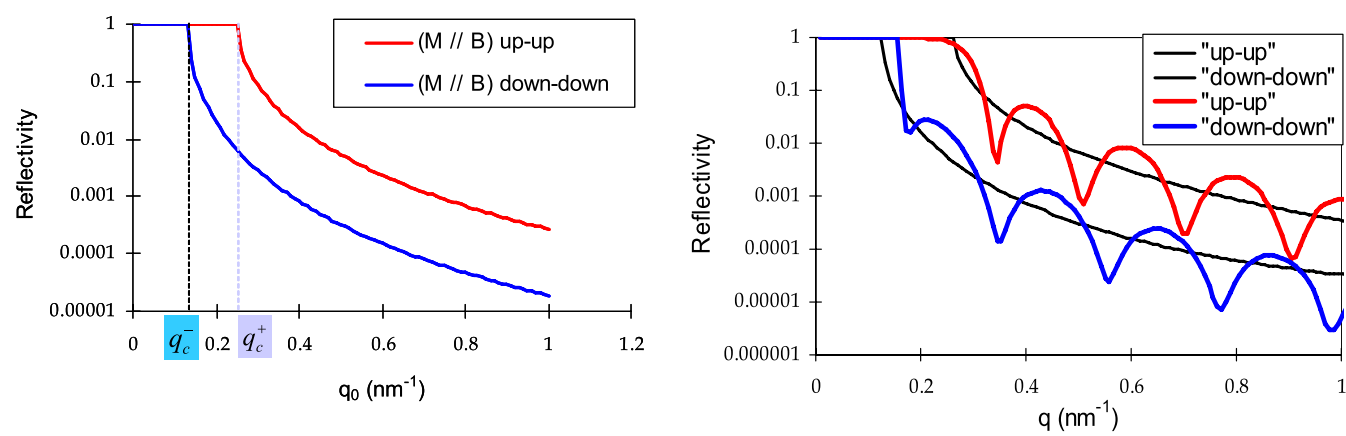

Figure 9. Reflection on a semi infinite magnetic medium; (b) reflection on a magnetic thin film ( $\mathrm{Fe}(30 \mathrm{~nm})$ on sapphire). The oscillations are directly related to the Fe film thickness.

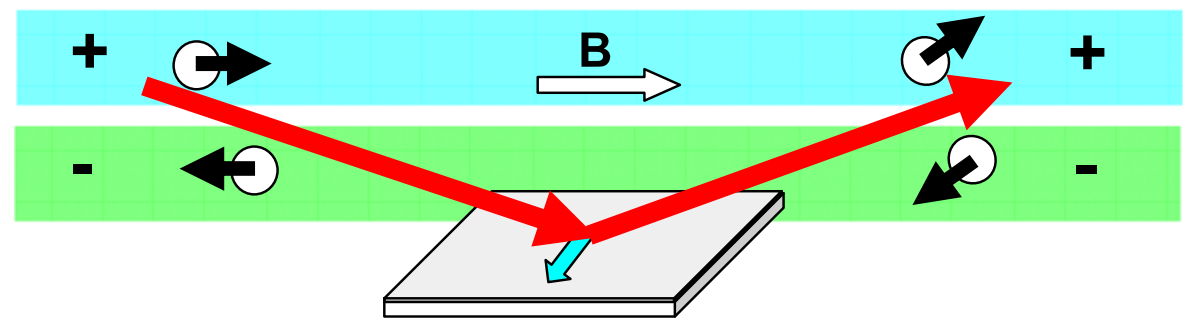

Figure 10. In the case of a magnetization perpendicular to the applied magnetic field, the neutrons do not remain in their eigenstate. It is possible to measure 4 different cross sections $R^{++}, R^{--}, R^{+-}, R^{-+}$.

The reflected amplitudes are given by:

$$
r^{++}=\frac{k_{\perp 0}-k_{\perp s}^{+}}{k_{\perp 0}+k_{\perp s}^{+}} \text {and } r^{--}=\frac{k_{\perp 0}-k_{\perp s}^{-}}{k_{\perp 0}+k_{\perp s}^{-}} .
$$

The reflected intensities are given by:

$$
R^{+}=\left|r^{++}\right|^{2} \text { and } R^{-}=\left|r^{--}\right|^{2} .
$$

The critical angle for "up" and "down" neutrons is given by:

$$
n^{+}=\cos \theta_{c}^{+} \text {and } n^{-}=\cos \theta_{c}^{-} .
$$

The reflection on a magnetic semi-infinite medium is very similar to the non magnetic case. The only difference is that the "up" and "down" curves are split. The splitting is directly related to the magnetization amplitude.

\subsubsection{Magnetization perpendicular to the applied magnetic field}

During reflection, the neutron will experience partial spin-flip (they precess around the magnetization during the reflection). The sample transfer matrix has the general form:

$$
\left(\begin{array}{ll}
R^{++} & R^{+-} \\
R^{-+} & R^{--}
\end{array}\right)
$$



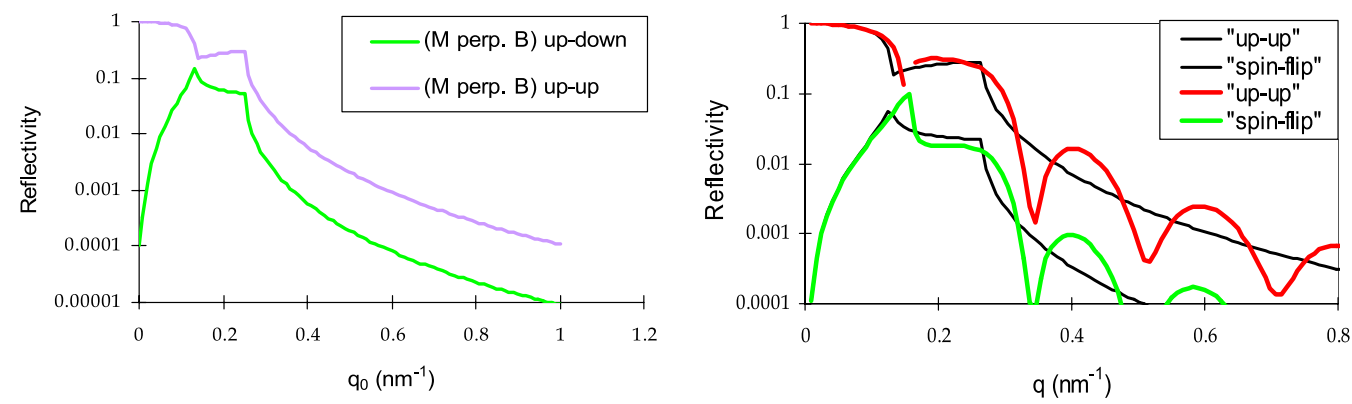

Figure 11. Reflection on a semi infinite magnetic medium where the guide field is perpendicular to the magnetization; (b) reflection on a magnetic thin film (Fe $(30 \mathrm{~nm})$ on sapphire). Extra modulations appear.

For an "up" (resp. "down") polarised beam, $\varphi=1|+\rangle$ (resp. $\varphi=1|-\rangle$ )

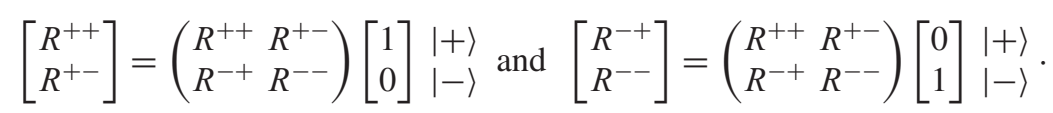

The reflected spin-flip intensity is given by:

$$
r^{+-}=\frac{1}{2} \frac{k_{\perp 0}\left(k_{\perp s}^{+}-k_{\perp s}^{-}\right)}{\left(k_{\perp 0}^{+}+k_{\perp s}^{-}\right)\left(k_{\perp 0}^{+}+k_{\perp s}^{-}\right)} .
$$

The "up-up" and "down-down" reflected intensities are equal and are the sum of three terms:

$$
R^{++}=R^{--}=\left|r^{2}\right|=\frac{1}{4}\left|r^{++}\right|^{2}+\frac{1}{4}\left|r^{--}\right|^{2}+\frac{1}{2} \operatorname{Re}\left(r^{++} \times r^{++}\right) .
$$

The first two terms corresponds to the intensities of the non-spin-flip signals in the case of a magnetization aligned with the external magnetic field; they are weighted by a coefficient. These 2 terms introduce two discontinuities at the positions $q_{c}^{+}$and $q_{c}^{-}$in the reflectivities (see Fig. 11). An interference term adds to this, whose expression is non trivial. It does not qualitatively change the shape of the curves.

The spin-flip intensity is given by:

$$
R^{+-}=R^{-+}=\left|r^{+-}\right|^{2}=\frac{1}{4}\left|\frac{k_{\perp 0}\left(k_{\perp s}^{+}-k_{\perp s}^{-}\right)}{\left(k_{\perp 0}^{+}+k_{\perp s}^{-}\right)\left(k_{\perp 0}^{+}+k_{\perp s}^{-}\right)}\right|^{2} .
$$

The characteristic shape of the spin-flip signal (see Fig. 13) is given by the term $\left|k_{\perp s}^{+}-k_{\perp s}^{-}\right|^{2}$.

In a simple picture, one can summarize by saying that the $R^{++}$and $R^{--}$cross sections give information about the magnetization components along the applied magnetic field. The spin-flip crosssections $R^{+-}=R^{-+}$give information about the magnetization component perpendicular to the applied field.

\subsection{Instrumentation}

In order to perform polarised neutron reflectivity experiments you simply need to add a few optical elements on your 2-axis spectrometer. You need a polarizer to polarise your beam and a flipper which allows you to manipulate your incident polarisation. The neutron beam is then reflected on the sample and you then use a symmetrical set-up to analyze the reflected polarisation. Nowadays, the polarisers and flippers are standard off-the shelf optical elements. 


\section{Guide field}

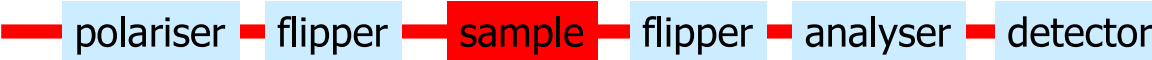

Figure 12. Elements required in order to perform a polarised neutron experiment.
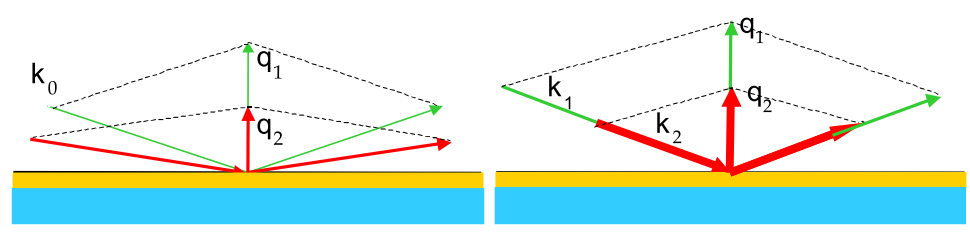

Figure 13. Angular scan $\theta / 2 \theta$; (b) time of flight scan.

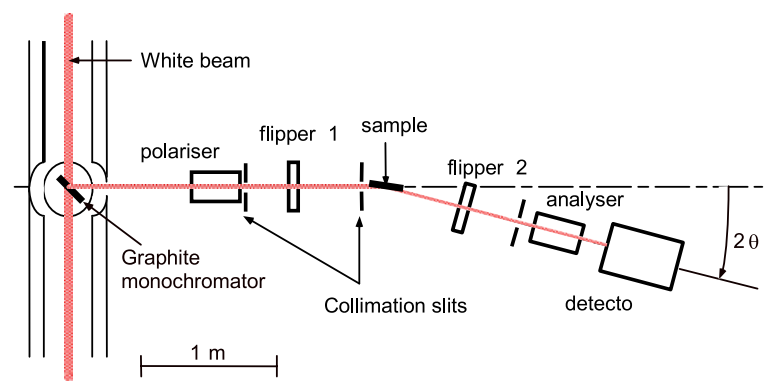

Figure 14. Classical 2 axis spectrometer (spectrometer PADA at the LLB).

At the moment, in all the existing polarised neutron reflectometers, one works with a small applied magnetic field $\mathbf{B}$ over the whole neutron path. It is possible to measure 4 cross-sections depending on the neutron polarisation with respect to the applied field.

There are two ways of varying the scattering wave-vector: $Q=4 \pi \sin \theta / \lambda$

- Classical angular scan $\theta / 2 \theta$. The reflectometer is using a single incident wavelength (see Fig. 13a)

- Time of Flight (ToF): the incident angle is kept fixed and the incident wavelength is varied (typically in the range of 0.2 to $2 \mathrm{~nm}$ ) (see Fig. 13b).

The ToF mode is convenient for the study of free liquid surfaces. It also allows to measure the whole reflectivity spectrum at the same time. The measuring time simply increases the statistics. A fixed wavelength reflectometer is a simple 2-axis instrument (see Fig. 14). The only requirement is that good collimations are available. Typical instruments work with a wavelength of 0.4 to $0.5 \mathrm{~nm}$. The requested angular divergence is of the order of $0.03^{\circ}(0.5 \mathrm{mrad})$.

A time-of-flight reflectometer is a slightly more complex instrument. It requires using a chopper on continuous sources. In the case of polarised neutrons, broadband polarisers and flippers are required. 


\section{Collection SFN}

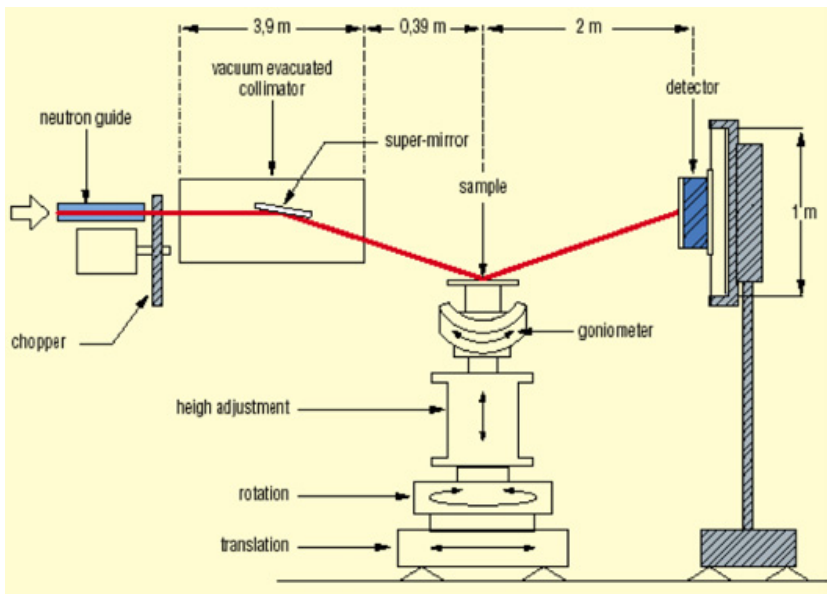

Figure 15. ToF spectrometer EROS at the LLB.

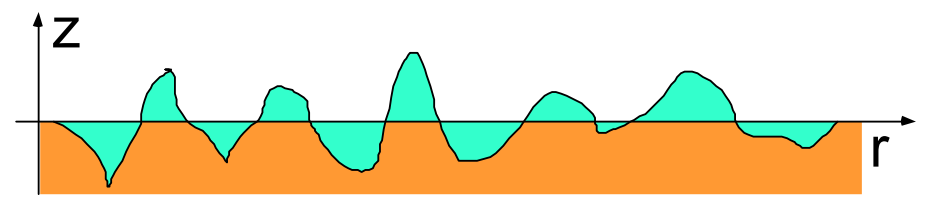

Figure 16. The interface between two materials is never perfect. The perturbation with respect to the perfect interface is represented in green.

\section{ROUGHNESS EFFECTS}

In real life, one has always to deal with imperfect systems. The layers are composed by atoms and the interfaces are never perfect. We have to distinguish 2 key parameters which will be of crucial importance with respect to their influence on the reflectivity. The first one is the averaged height of the fluctuations $\sigma$ which are characterized by:

$$
\sigma=\sqrt{\left\langle z^{2}\right\rangle_{s}}
$$

The second length-scale is given by $\xi$ which characterizes the "in-plane" fluctuations length-scales.

In practice, we can distinguish 3 classes of roughness:

- Roughness at the atomic level: which corresponds to the inter-diffusion of atoms between the thin films, usually $\sigma$ is of the order of $0.5-1 \mathrm{~nm}$ and $\xi \leq 100 \mathrm{~nm}$. In this case, the measured specular signal cannot "see" these details and the system can be approximated by a smooth profile (see Fig. 17a).

- Intermediate roughness: $\xi$ from $0.1 \mu \mathrm{m}$ to $50 \mu \mathrm{m}$ and $\sigma \sim 1 \mathrm{~nm}$.

In this case, a part of the signal scattered by the surface is not scattered in the specular direction. The specular reflectivity signal must be corrected by an attenuation factor called the Nevot-Croce factor: $e^{-\sigma^{2} q^{2}}$.

The signal scattered off the specular direction can also be measured and characterized and will give detailed information about the in-plane structure of the interface.

- A large scale roughness: $\xi \geq 100 \mu \mathrm{m}$. 
(a)

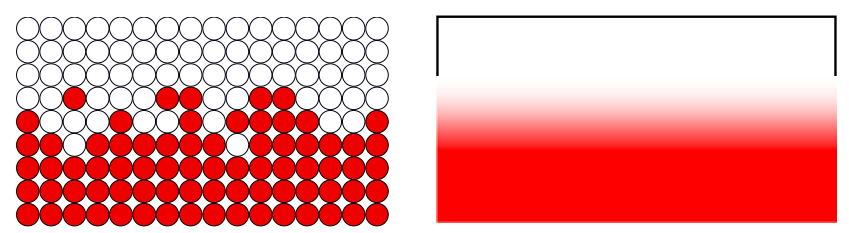

(b)

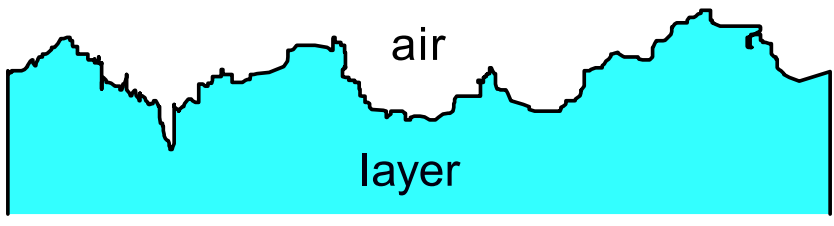

(c)

substrate

Figure 17. The atomic inter-diffusion between 2 layers; the details cannot be observed in a reflectivity geometry (b) the roughness at a micrometric level. (c) bending of the substrate or fluctuating layer thickness.
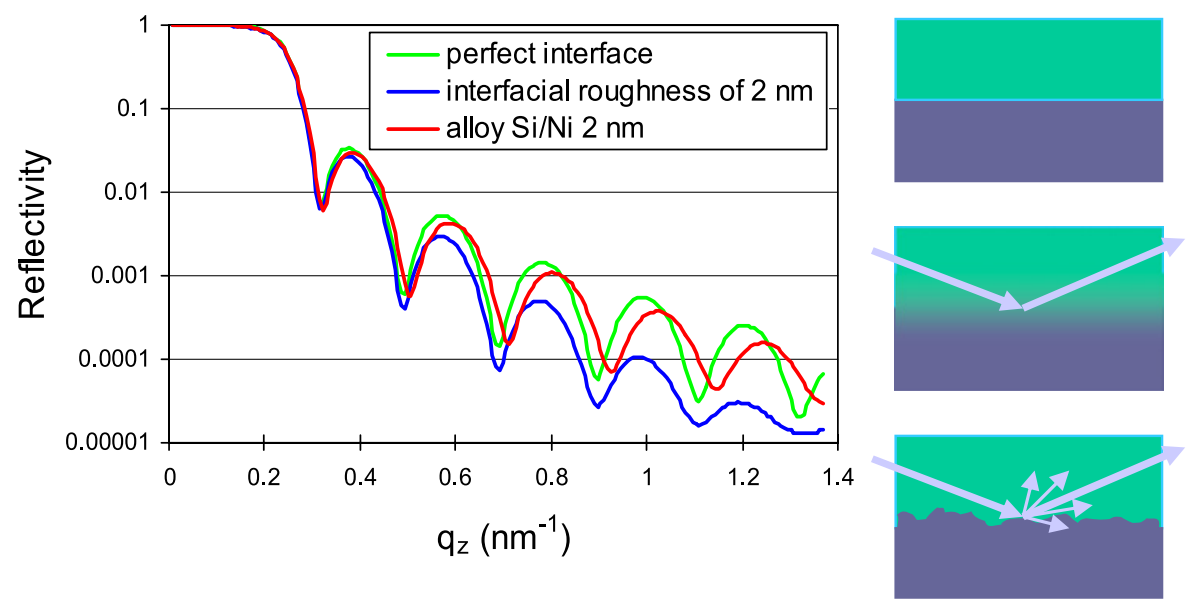

Figure 18. Influence of the roughness on the reflectivity. (green) perfect interface, (red) smooth interface due to atomic inter-diffusion (blue) "rough" interface, some neutrons are scattered "off" the specular direction and thus the signal decays faster at large $Q$ values.

These fluctuations usually correspond to a "non-flatness" of the substrate or to big inhomogeneities of the layer thickness. In this case, the easiest way to process the data is to consider that the incidence divergence is increased (sample waviness). One should avoid this situation since most of the reflectivity information is dramatically blurred by such an effect.

The effect of the different types of roughness is compared on Figure 18. In the case of some atomic interdiffusion (red), the shape of the signal is very similar to the perfect system except that the "effective" thickness of the top layer is smaller and thus the oscillations are shifted. In the case of the "rough" interface, some neutrons are scattered "off" the specular direction and thus the signal decays faster at large $\mathrm{Q}$ values. One has to apply the Nevot-Croce factor to account for this decay. 


\section{Collection SFN}
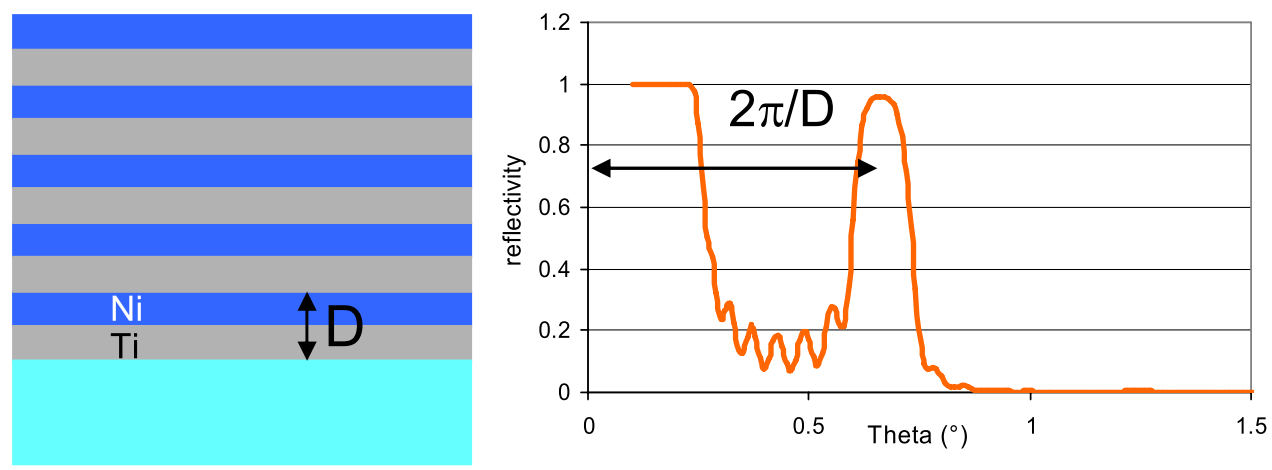

Figure 19. (a) Multilayer $[\mathrm{Ni}(5 \mathrm{~nm}) / \operatorname{Ti}(5 \mathrm{~nm})]_{10}$ deposited on glass. (b) Reflectivity of the multilayer. Below the critical angle there is total reflection; around $\theta=0.7^{\circ}$ there is a diffraction peak corresponding to the bilayer thickness D. The reflectivity can be very high (close to $95 \%$ ).
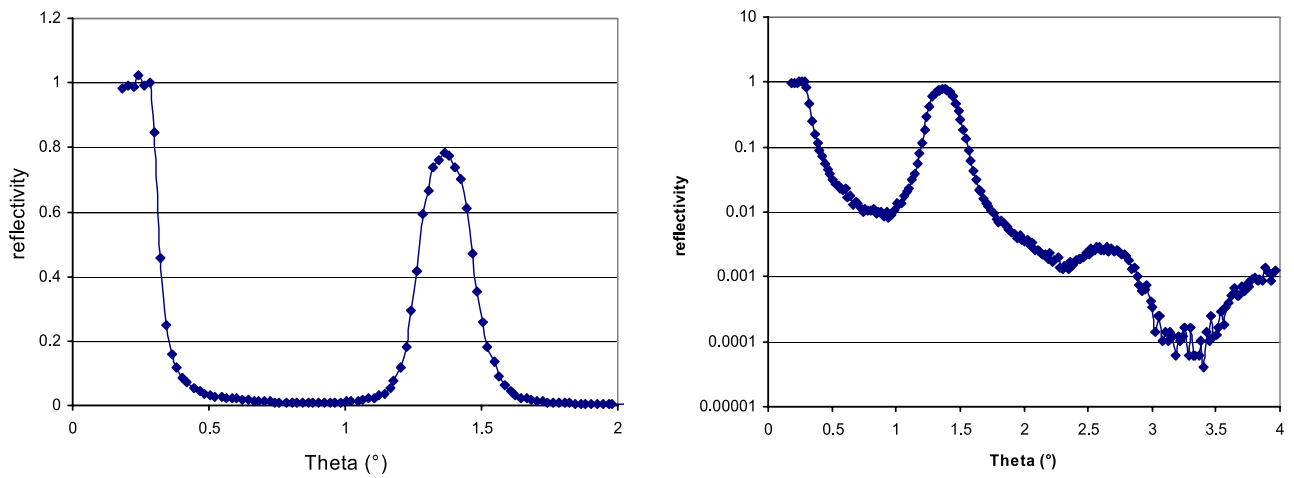

Figure 20. Reflectivity of monochromators produced by Swiss Neutronics (left) linear scale; (right) log scale, higher harmonics can be measured.

\section{EXAMPLES}

In this chapter we will illustrate the possibilities offered by the technique of polarized neutron reflectometry (PNR). The technique can be applied on various types of systems:

- Thin films: oxide layers (manganites, magnetite)

- Multilayers (GMR systems)

- Superlattices: Rare-earth super-lattices (Y, Dy, Er, Tb), magnetic semi-conductors.

\subsection{Multilayer monochromators}

The thin film technology allows to deposit very thin layers of materials on substrates. It is thus possible to produce artificial 1D crystals with periodicities down to $5 \mathrm{~nm}$ (see Figure 19). This allows to produce systems which behave as monochromators.

Such structures are now commonly produced over large surfaces $\sim \mathrm{m}$ to produce neutron monochromators. Figure 20 shows the reflectivity of a such a monochromator produced by Swiss Neutronics. The period of the bilayer is $9 \mathrm{~nm}$. The reflectivity of this monochromator reaches $80 \%$. It is possible to tune the monochromatization band by adjusting the period of the bilayers.

Measurements in the direct space by TEM show the quality of the interfaces of such metallic multilayers. 


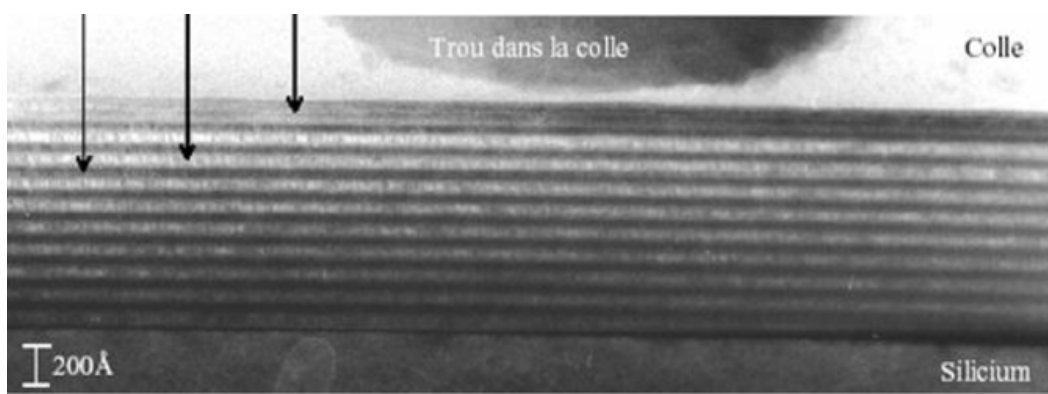

Figure 21. HRTEM image in the direct space of such a multilayer system.
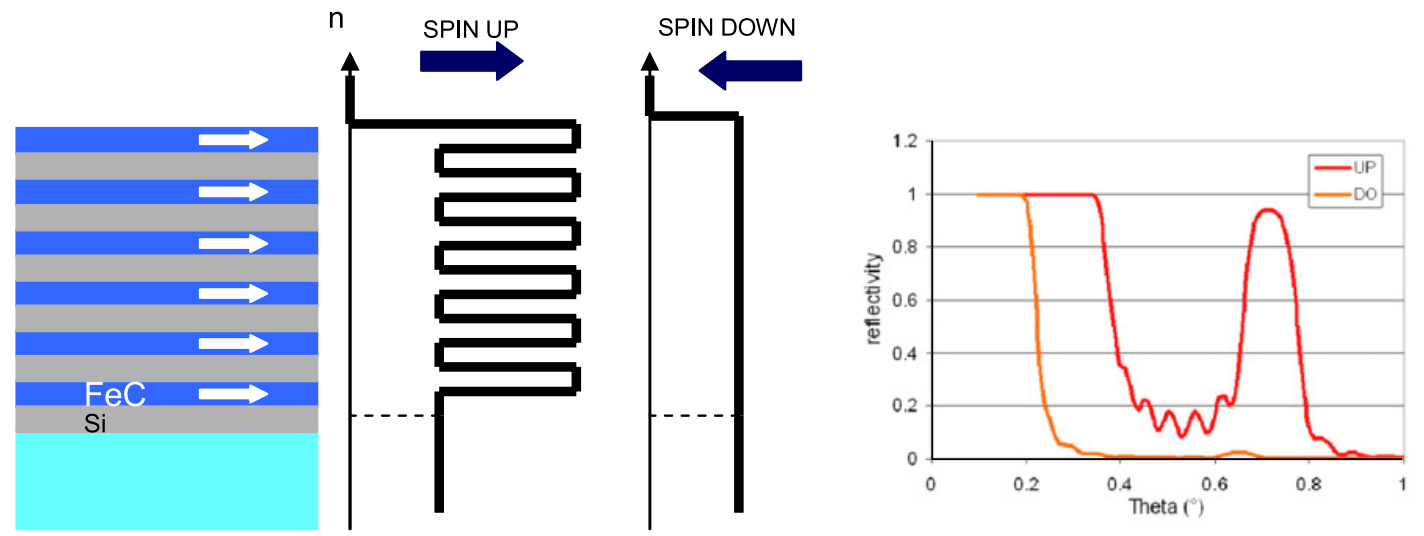

Figure 22. Multilayer $[\mathrm{FeCo} / \mathrm{Si}]_{10}$ deposited on glass. (b) The interaction potential as a function of the polarisation. (c) Reflectivity of the multilayer.

\subsection{Neutron polarizers}

It is possible to introduce magnetic materials in multilayer systems. ${ }^{1}$ In order to produce efficient optical elements, high optical index and high optical contrast is required. In order to maximize the magnetic contrast, $\mathrm{Fe}$ or FeCo alloys are used. The spacer is a material close to silicon because its optical index matches the $n^{-}$optical index of the magnetic layer. When the neutrons are polarized "up", they see a strong optical modulation (see Fig. 22b). When the neutrons are polarized "down", they see a smooth interaction potential and no diffraction occurs. Such a device behaves as a polarizing monochromator.

Since the neutron absorption is very low, it is possible to stack multilayers of varying periods in order to superimpose a series of diffraction peaks. It is thus possible to "fill" the whole range between the total reflection angle and a $Q^{\max }$ value and to obtain an artificially high optical index mirror. The $Q^{\max }$ value is limited by the thinnest layers than can be deposited (presently of the order of $5 \mathrm{~nm}$ ). This allows to produce mirrors that have an artificial optical index which is 3 to 4 times larger than Ni.

The following Figure 23 shows the reflectivity of a real polarizer (produced by the ILL). Below $\mathrm{Q}=0.15 \mathrm{~nm}^{-1}$, both spin states are reflected. Above $0.15 \mathrm{~nm}^{-1}$, the "down" spin state is not reflected; up to $0.6 \mathrm{~nm}^{-1}$, the "up" spin state is reflected. Figure $25 \mathrm{~b}$ shows the flipping ratio "up"/"down", it reaches up to 150 , corresponding to a beam polarization above $99.3 \%$.

\footnotetext{
${ }^{1}$ In the previous example, elements such as Carbon were added in the Ni so that there was no magnetic signal.
} 


\section{Collection SFN}
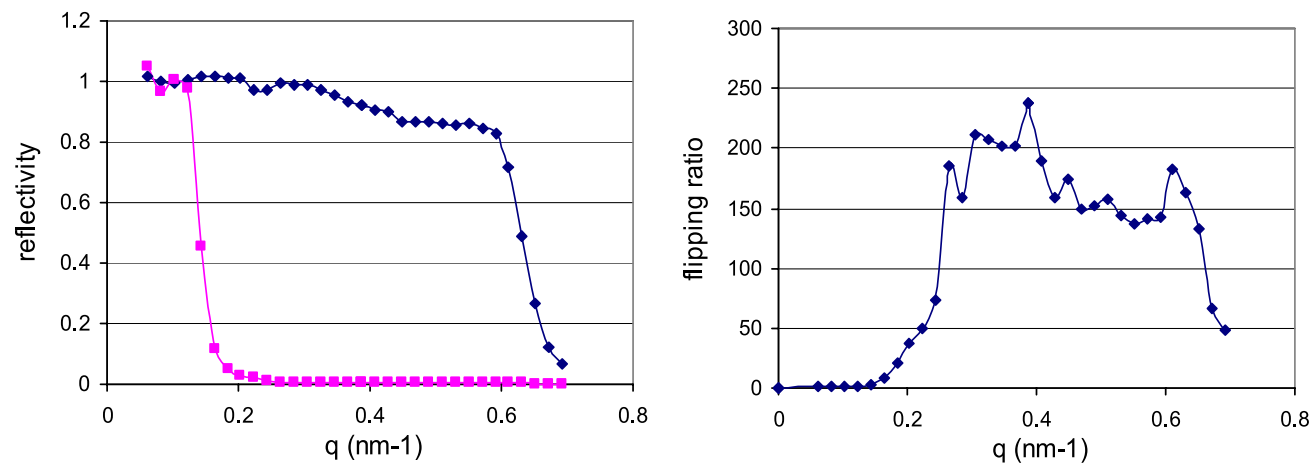

Figure 23. Reflectivity of a polarizing super-mirror.

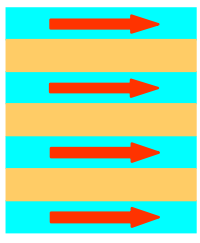

$\mathrm{J}_{1}>0$

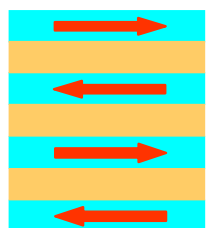

$\mathrm{J}_{1}<0$

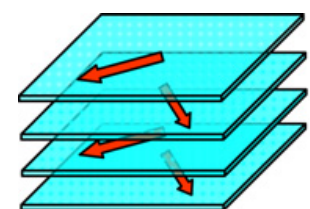

$\mathrm{J}_{1}>0$ and $\mathrm{J}_{2}<0$

Figure 24. (a) Ferromagnetic coupling; (b) anti-ferromagnetic coupling; (c) quadratic coupling.
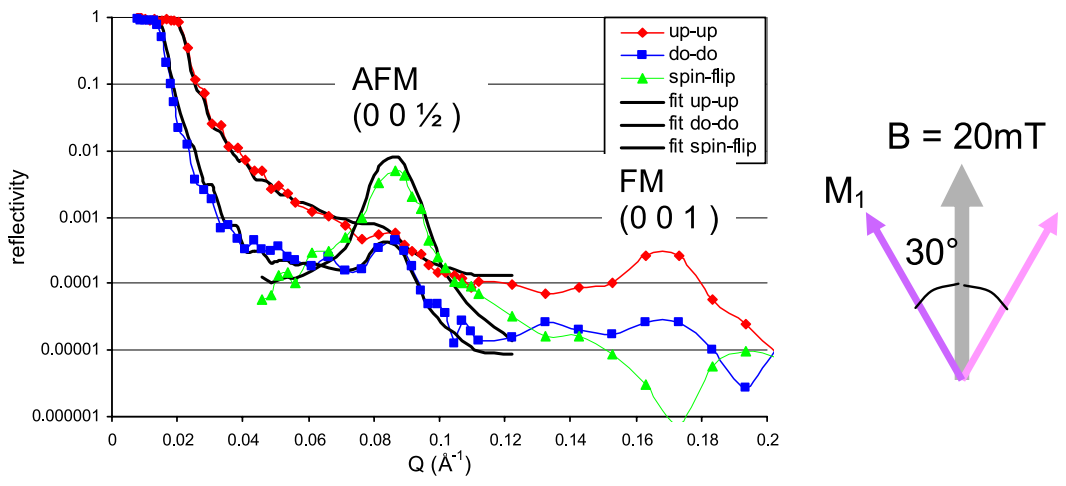

Figure 25. Reflectivity of a $[\mathrm{Fe}(2.4) / \mathrm{Si}(1.2)]_{n}$ measured at $5 \mathrm{~K}$.

\subsection{Magnetic multilayers}

We have previously shown technological elements. In a more general way, PNR can be used to study the magnetic configuration of a multilayer system. It allows to:

- access to the magnetization amplitude and direction in each layer, i.e. determine in-depth magnetic profiles.

- measure the absolute magnetic moment in Bohr magneton per formula units.

The depth resolution is of the order of $2-3 \mathrm{~nm}$ in simple systems. PNR is a surface technique and thus is not sensitive to paramagnetic or diamagnetic contributions from the substrate. There is no absorption. There are no phenomenological parameters. The data are "naturally" normalized. 

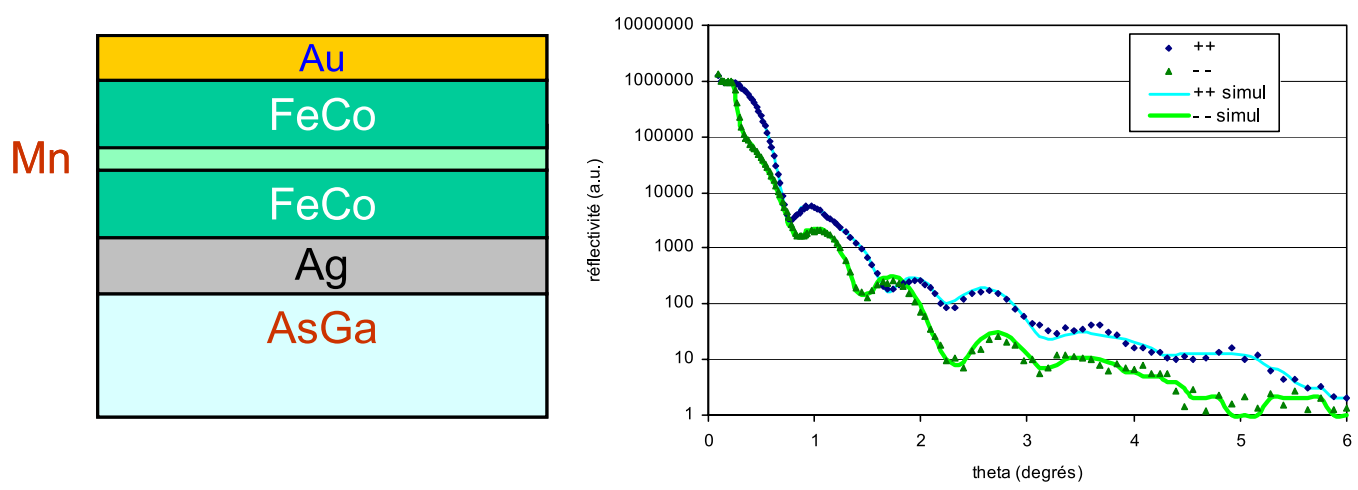

Figure 26. (a) $\mathrm{Fe} / \mathrm{Mn} / \mathrm{Fe}$ trilayer system; (b) reflectivity in the saturated state.

\subsubsection{Magnetic coupling}

In magnetic multilayers where magnetic layers are separated by a non magnetic spacer, different types of coupling can appear. The coupling energy can be phenomenologically described by a coupling energy:

$$
E_{\text {coupling }}=-J_{1} \vec{S}_{1} \cdot \vec{S}_{2}-J_{2}\left(\vec{S}_{1} \cdot \vec{S}_{2}\right)^{2}
$$

\subsubsection{Exchange coupling in multilayers}

We present here the example of $[\mathrm{Fe} / \mathrm{Si}]_{n}$ multilayers produced by K. Fronc from the Polish. Acad. Science. The system presented is $\mathrm{GaAs} / /[\mathrm{Fe}(2.4 \mathrm{~nm}) \mathrm{Si}(1.2 \mathrm{~nm})]_{20}$. From the PNR measurements, it was possible to determine that at room temperature, a magnetic AF order sets in. When the temperature is decreased, a more complex magnetic order sets in. On the reflectivity scans (see Fig. 25), one can observe different features. The first one is the (001) peak of the super-lattice structure which is reminiscent of the chemical structure of the system. The second important feature is the peak observed at the (001/2) position. This is a peak of purely magnetic origin. It indicates that an anti-ferromagnetic coupling exists between adjacent Fe layers.

Even more interesting is that when the temperature is decreased below $200 \mathrm{~K}$, at the antiferromagnetic position, the spin-flip peak becomes dominant. This means that the coupling between the layers is not simply anti-ferromagnetic anymore. A numerical modeling suggests that the magnetizations of adjacent Fe layers make an angle of $30^{\circ}$ with respect to the applied field.

\subsubsection{Magnetic single layers}

PNR allows to probe the magnetization of very thin systems. The magnetic behaviour of $\mathrm{Fe} / \mathrm{Mn} / \mathrm{Fe}$ has been studied. The typical structure of the sample is shown on Figure 29a. The "active" region is formed by the layers $\mathrm{Fe} / \mathrm{Mn} / \mathrm{Fe}$. The $\mathrm{Ag}$ layer is used to promote an epitaxial growth of the system. The Au layer is a simple protective capping. The presented system is $\mathrm{Fe}_{0.5} \mathrm{Co}_{0.5} / \mathrm{Mn}\left(8 \mathrm{~A}^{\circ}\right) / \mathrm{Fe}_{0.5} \mathrm{Co}_{0.5}$. The specificity of this system is that the magnetic coupling between $\mathrm{Fe} / \mathrm{Mn}$ and $\mathrm{Co} / \mathrm{Mn}$ are of opposite sign. This leads to a complex magnetic behaviour of the Mn layer.

A first measurement was performed in a saturating field (see Fig. 26b). A numerical modeling of these data shows that the magnetic moment in the $\mathrm{Fe}_{0.5} \mathrm{Co}_{0.5}$. layers is $2.4 \mu \mathrm{B} /$ atom (as in bulk materials). A net magnetic moment of $0.8 \mu \mathrm{B} /$ atom in $\mathrm{Mn}$ is also observed. Such an induced magnetization was theoretically predicted for FeCo alloys. In similar systems without Co, no magnetic moment is observed in the Mn layer. 


\section{Collection SFN}
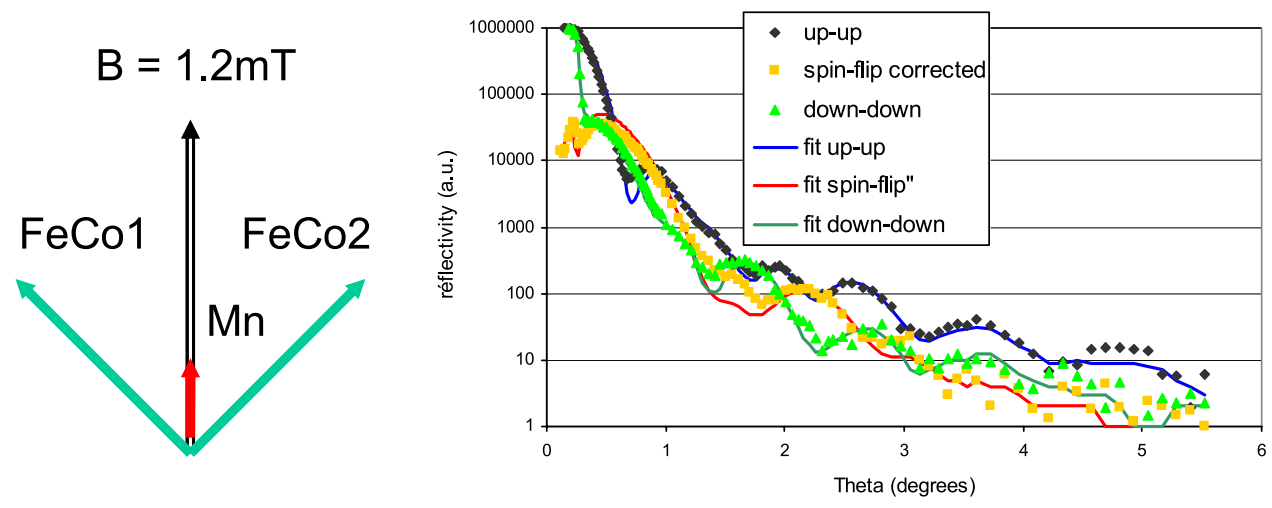

Figure 27. (a) Reflectivity in the remanent state; (b) magnetic configuration as deduced from the fit.
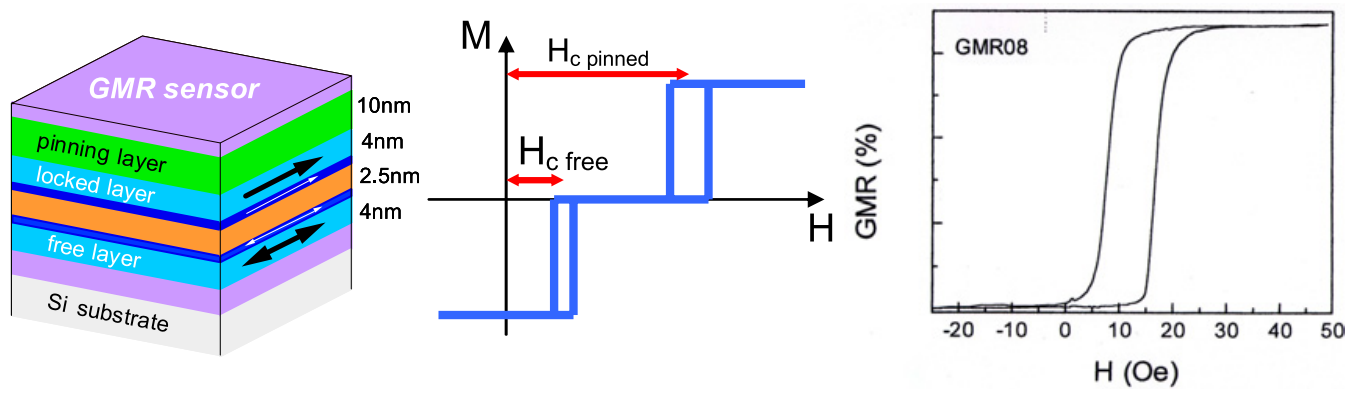

Figure 28. (a) Typical GMR system; (b) hysteresis cycle on such a system; (c) resistivity of the GMR system.

The applied field was then decreased down to $1.2 \mathrm{mT}$. The reflectivity was remeasured. In these conditions a large spin-flip signal is observed (see Fig. 27b). The reflectivity data was fitted by letting the magnetization directions vary. The best adjustment is obtained when the magnetization of the layers make an angle of $45^{\circ}$ with respect to the applied field. The 2 magnetic layers make an angle of $90^{\circ}$. We have a quadratic coupling.

\subsubsection{GMR sensors}

In the last 15 years, a large effort has been devoted to the fabrication and optimization of Giant MagnetoResistive Sensor (GMR) which consist of tri-layer magnetic systems: 2 magnetic layers (Permalloy) are separated by a non magnetic spacer. Such sensors are used in magnetic recording because the electrical resistivity depends on the relative orientation of the 2 permalloy layers. A pinning layer which is usually an anti-ferromagnet such as FeMn locks the magnetisation of the top permalloy layer. The magnetization of second permalloy layer can freely rotate in the plane of the layer. The resistivity of such a system depends on the relative orientation of the 2 magnetic layers. By following the resistivity of the system you can measure the applied field and use these devices as very sensitive field sensors.

A typical GMR system is presented on Figure $28, \mathrm{SiO}_{2} / / \mathrm{Ta} / \mathrm{NiFe} / \mathrm{CoFe} / \mathrm{Cu} / \mathrm{CoFe} / \mathrm{MnPt} / \mathrm{Ta}$. This structure is used for read heads in high density tape recording. The aim of the study was to understand 


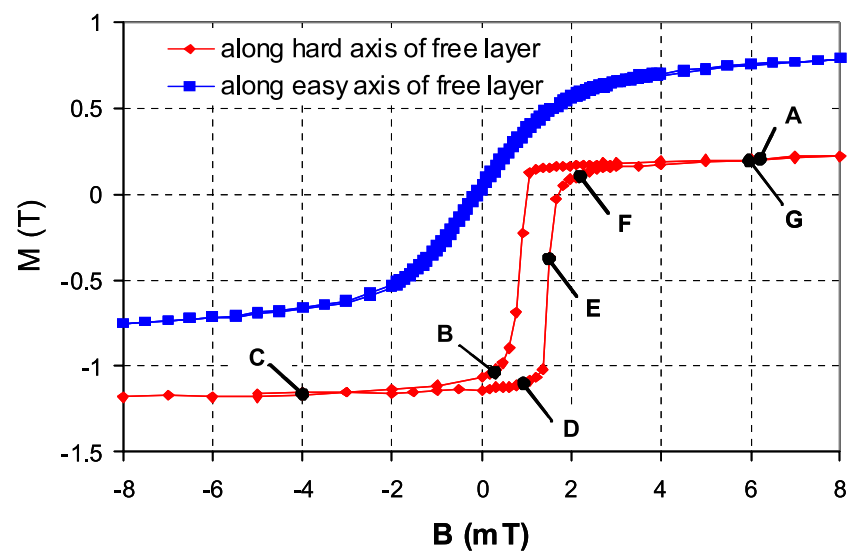

Figure 29. Hysteresis cycle of the multilayer.
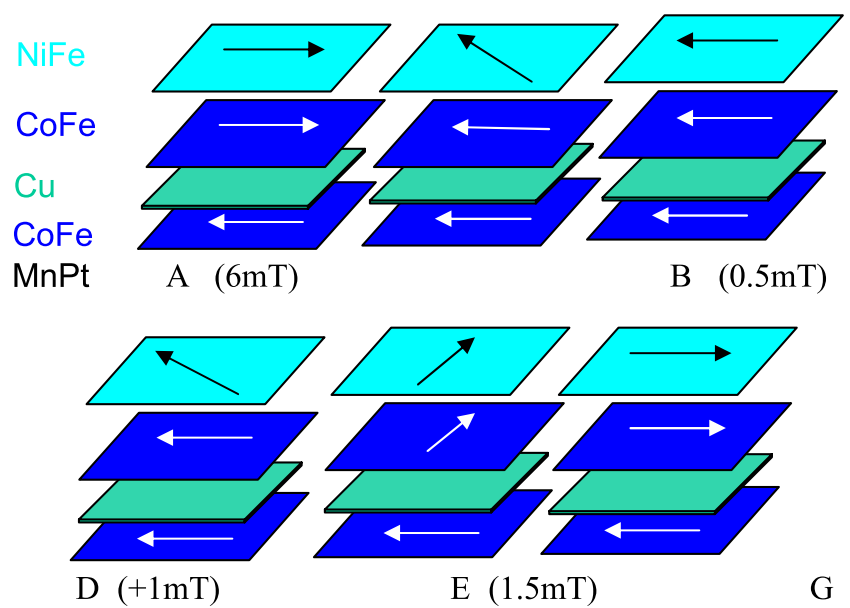

Figure 30. Evolution of the magnetic configuration of the GMR as a function of the applied field.

and optimize the magnetic behaviour of the different layers so as to have a system with a little magnetic noise as possible, so that the system is more performing. PNR can be used to follow the behaviour of the different layers of the sensor as a function of the applied field. The Figure 29 shows the magnetization measurements on the GMR system. The easy axis of the AF layer is perpendicular to the easy axis of the free layer.

The PNR was followed as a function of the applied field (see points on Fig. 29). The magnetic configuration could then be followed along the hysteresis cycle and it was possible to reconstruct the process of reversal of the magnetic layers and especially of the soft magnetic layer. Starting from the anti-parallel configuration, just after this first reversal when the field is decrease, one can observe that the magnetisation of the Permalloy layer has not fully flipped but that there is actually a rotation gradient inside the soft permalloy layer. One side, the permalloy layer is constrained by a thin CoFe layer but there is a competition with a magnetic anisotropy which has been introduced during the deposition 


\section{Collection SFN}

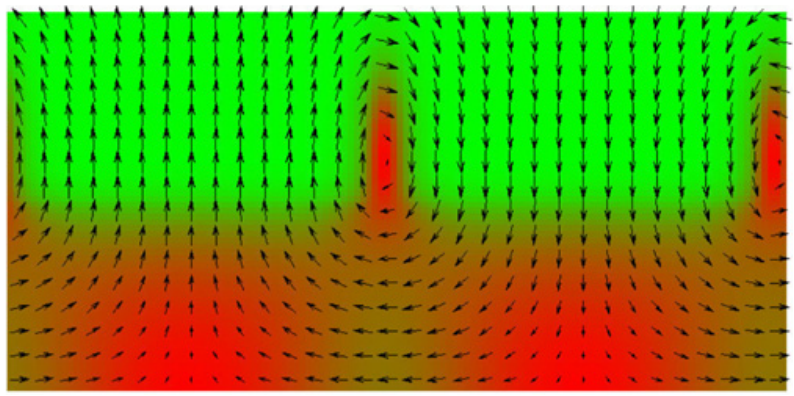

Figure 31. Calculated micro-magnetic configuration in a FePd $(30 \mathrm{~nm}) / \mathrm{FePd}(30 \mathrm{~nm})$ bilayer (side view). The FePd layer with a perpendicular anisotropy induces the formation of magnetic domains.

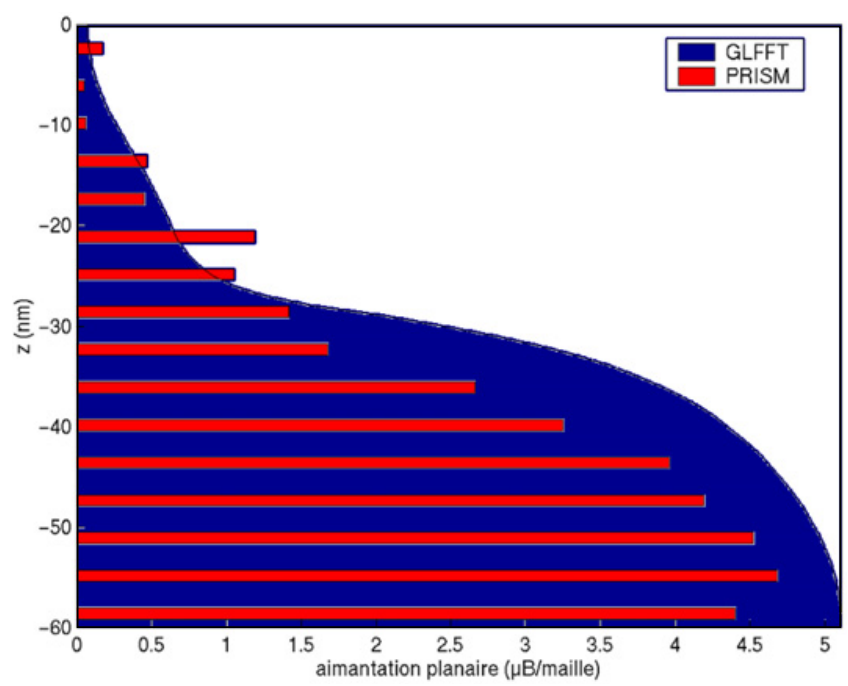

Figure 32. Comparison of the magnetization profile as deduced from the micro-magnetic calculation and from the neutron data modeling. The agreement is very satisfactory.

process and which is at $90^{\circ}$ with respect to this direction. The rotation gradient through the permalloy layer reached $25^{\circ}$ from top to bottom.

This rotation is very important because it is the reason why the magnetic rotation of the different layer is coherent that is without magnetic domains formation and this is especially important in terms of magnetic noise in the system. On the way back one observes the same phenomenon.

\subsubsection{Magnetic domains}

It is possible to characterize continuous profiles by PNR. In FePd layers, complex magnetic structures appear. G. Beutier et al from CEA/DRFMC Grenoble have studied systems such as FePd/FePd bi-layers in which one layer has an in-plane anisotropy and the second layer has a perpendicular anisotropy (see Fig. 31). 


\section{JDN 20}

Using PNR it is possible to deduce the magnetization profile through the depth of the system (see Fig. 32). The PNR measurements only provide the average in-plane magnetic moment as a function of the depth in the film. The agreement between the calculation and the measurement is very satisfactory.

\section{CONCLUSION}

We have presented different possibilities of the use of polarized neutron reflectometry for the study of thin film systems. The studies were limited to a characterization of the in-depth magnetic profile of multilayers. It is possible to extend the studies to characterize the in-plane structure of multilayer systems using "off-specular" scattering and Grazing Incidence SANS. 